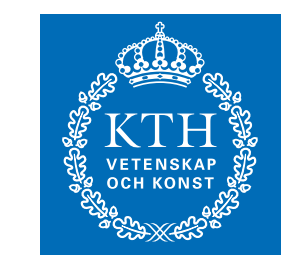

KTH Electrical Engineering

\title{
Post-User-Selection Quantization and Estimation of Correlated Frobenius and Spectral Channel Norms
}

\author{
Invited Paper \\ Proceedings of IEEE International Symposium on Personal, Indoor and \\ Mobile Radio Communications (PIMRC)
}

September 15-18, Cannes, France, 2008

(C) 2008 IEEE. Published in the IEEE 2008 International Symposium on Personal, Indoor and Mobile Radio Communications (PIMRC 2008), scheduled for September 15-18, 2008 in Cannes, France. Personal use of this material is permitted. However, permission to reprint/republish this material for advertising

or promotional purposes or for creating new collective works for resale or redistribution to servers or lists, or to reuse any copyrighted component of this work in other works, must be obtained from the IEEE. Contact: Manager, Copyrights and Permissions / IEEE Service Center / 445 Hoes Lane / P.O. Box 1331 / Piscataway, NJ 08855-1331, USA. Telephone: + Intl. 908-562-3966.

\section{EMIL BJÖRNSON AND BJÖRN OTTERSTEN}

Stockholm 2008

\author{
KTH Royal Institute of Technology \\ ACCESS Linnaeus Center \\ Signal Processing Lab
}

IR-EE-SB 2008:039, Revised version with minor corrections 


\title{
Post-User-Selection Quantization and Estimation of Correlated Frobenius and Spectral Channel Norms
}

\author{
(Invited Paper) \\ Emil Björnson and Björn Ottersten \\ ACCESS Linnaeus Center \\ Signal Processing Lab \\ Royal Institute of Technology (KTH) \\ SE-100 44 Stockholm, Sweden \\ Email: emil.bjornson@ee.kth.se
}

\begin{abstract}
This paper considers quantization and exact minimum mean square error (MMSE) estimation of the squared Frobenius norm and the squared spectral norm of a Rayleigh fading multiple-input multiple-output (MIMO) channel with onesided spatial correlation. The Frobenius and spectral norms are of great importance when describing the achievable capacity of many wireless communication systems; in particularly, they correspond to the signal-to-noise ratio (SNR) of space-time block coded and maximum ratio combining transmissions, respectively. Herein, a general quantization framework is presented, where the quantization levels are determined to maximize the feedback entropy. Quantization based on the post-user-selection distribution is discussed, and analyzed for a specific scheduler. Finally, exact results on MMSE estimation of the capacity and the SNR, conditioned on a quantized channel norm, are presented.
\end{abstract}

\section{INTRODUCTION}

Fading is a major challenge in establishing reliable wireless communication systems. The use of multiple antennas at both the transmitter and the receiver side, so-called multipleinput multiple-output (MIMO), show promising capabilities of mitigating fading and exploiting the gains of multipath signals by introducing diversity. When full channel state information (CSI) is available at both the transmitter and the receiver side, the throughput can even increase linearly with the number of antennas in rich scattering environments [1], [2]. Unfortunately, full CSI is not automatically available in most systems, but needs to be estimated at the receiver [3] and then fed back with finite precision. Thus, in many fast-fading environments it is only reasonable to assume that statistical CSI (which varies quite slowly) is available at the transmitter, and possibly a recurring feedback that describes the current channel using a limited number of bits.

Because the time spent on acquiring CSI at the transmitter reduces the actual transmission of data, the design of transmission techniques that provide good performance using only a limited CSI of feedback is of great importance. Two such approaches are orthogonal space-time block codes (OSTBCs) [4], [5] and maximum ratio transmission (MRT) [6], [7]. OSTBCs are a clever way of transmitting several simultaneous

This work is supported in part by the FP6 project Cooperative and Opportunistic Communications in Wireless Networks (COOPCOM), Project Number: FP6-033533. data symbols over a block of symbol slots, such that the detection of them can be decomposed into independent and simple detection problems. Full CSI is required at the receiver, but this technique can, without any feedback, achieve full spatial diversity since all symbols are spread in all spatial directions. As a consequence, the effective channel gain will be the average gain over all spatial directions [5], which is more reliable than just choosing a random direction. In other words, the channel gain is proportional to the average of all squared singular values of the channel matrix, or similarly to the squared Frobenius norm of the channel.

MRT is based on beamforming, which means that a single data symbol is transmitted into the spatial direction that provides the strongest instantaneous channel gain. The receiver combines the signals received over its antennas to achieve this gain. In comparison with OSTBCs, scheduled users are also required to feed back the strongest beamforming direction [7]. However, the antenna diversity provided by this feedback gives a channel gain that is equal to the largest squared singular value, also known as the squared spectral norm of the channel, which may be considerably larger than the average value.

In order to achieve the capacity of OSTBCs or MRT, the transmitter needs the instantaneous channel gain to perform reliable transmission-rate adaptation, user-selection, and to estimate, for example, the signal-to-noise ratio (SNR) and the channel capacity. This gain information can be obtained by feedback. In this paper, we study the problem of quantizing the squared Frobenius norm and squared spectral norm for the purpose of entropy-maximizing feedback. We present a general framework that quantizes the variable into an interval, without forcing it to represent a specific value. The best representative of the interval depends on the application (e.g., SNR or capacity estimation).

In Section III, it is shown how to perform quantization that maximizes the entropy of an arbitrary function of the norm (e.g., the SNR or capacity). We also discuss how to incorporate the post-user-selection distribution of the norm into the quantization (i.e., the fact that strong users are more likely to be scheduled). In Section IV, we provide exact minimum mean square error (MMSE) estimators of the SNR and the capacity in Rayleigh fading systems with one-sided 
spatial correlation and feedback of the squared channel norm (either the Frobenius or the spectral norm, depending on the application). Numerical results are given in Section V and conclusions are drawn in Section VI. Due to the space limitation, only outlines of the proofs are given herein.

\section{A. Preliminaries}

For notational convenience we use boldface (lower case) for column vectors, $\mathbf{x}$, and (upper case) for matrices, $\mathbf{X}$. The transpose and conjugate transpose are denoted $[\cdot]^{T}$ and $[\cdot]^{H}$, respectively. If the $i j$ th element of $\mathbf{X}$ is $x_{i j}$, then $[\mathbf{X}]_{i j}=x_{i j}$. The Frobenius norm of $\mathbf{X}$ is $\|\mathbf{X}\|_{F}^{2} \triangleq \sum_{i} \sum_{j}\left|x_{i j}\right|^{2}$, where $\triangleq$ indicates a definition. Similarly, the squared spectral norm (i.e., induced 2-norm) is denoted $\|\mathbf{X}\|_{2}^{2}$ and is defined as the largest eigenvalue of $\mathbf{X}^{H} \mathbf{X}$ (i.e., the largest squared singular value of $\mathbf{X})$. The Heaviside step function, denoted $H_{A}(x)$, is 1 if $x \geq A$ and 0 otherwise. $\mathbb{R}^{+}$and $\mathbb{Z}^{+}$denote the sets of real-valued and non-negative integers numbers, respectively.

\section{System ModeL}

Consider the downlink of a communication system with a single base station, with an array of $n_{T}$ antennas, and $N \geq 1$ mobile users, each with an array of $n_{R}$ antennas. The symbolsampled complex baseband equivalent of the narrowband flat fading channel to user $k$ is represented by $\mathbf{H}_{k} \in \mathbb{C}^{n_{R} \times n_{T}}$, where the $i j$ th element describes the current channel gain from the $j$ th transmit antenna to the $i$ th receive antenna. These elements are modeled as Rayleigh fading with one-sided spatial correlation; that is, we assume that

$$
\mathbf{H}_{k}=\mathbf{R}_{\mathrm{Rx}, k}^{\frac{1}{2}} \widetilde{\mathbf{H}}_{k} \mathbf{R}_{\mathrm{Tx}, k}^{\frac{1}{2}},
$$

where either the receive correlation matrix $\mathbf{R}_{\mathrm{Rx}, k} \in \mathbb{C}^{n_{R} \times n_{R}}$ or the transmit correlation $\mathbf{R}_{\mathrm{Tx}, k} \in \mathbb{C}^{n_{T} \times n_{T}}$ has distinct eigenvalues, while the other correlation matrix is the identity matrix. The matrix $\widetilde{\mathbf{H}}_{k} \in \mathbb{C}^{n_{R} \times n_{T}}$ consists of independent and identically distributed complex Gaussian elements as $\mathcal{C N}(0,1)$. This kind of one-sided correlation appears in, for example, metropolitan environments where the base station is elevated and therefore is affected by just a few scatterers, while mobile users are surrounded by rich scattering [8]. In general, the amount of spatial correlation at an antenna array decreases with the antenna spacing and increasing number of scatterers in the immediate surroundings.

The received vector $\mathbf{y}_{k}(t) \in \mathbb{C}^{n_{R}}$ of user $k$ at transmission slot $t$ is modeled as

$$
\mathbf{y}_{k}(t)=\mathbf{H}_{k} \mathbf{x}(t)+\mathbf{n}_{k},
$$

where $\mathbf{x}(t) \in \mathbb{C}^{n_{T}}$ is the transmitted signal vector and $\mathbf{n}_{k}$ is additive white Gaussian noise. For simplicity, the average power has been normalized such that $E\left\{\|\mathbf{x}(t)\|_{F}^{2}\right\}=1$, where the average is taken over data symbols, and $\mathbf{n}_{k} \in \mathcal{C N}\left(\mathbf{0}, \mathbf{I}_{n_{T}}\right)$.

For OSTBC transmission, we use a code with rate $\frac{K}{T}$ to transmit $K$ data symbols $\mathbf{s}=\left[s_{1}, \ldots, s_{K}\right]^{T}$ over $T \geq K$ transmission slots. The code is represented by a matrix $\mathbf{C}(\mathbf{s}) \in$ $\mathbb{C}^{n_{T} \times T}$ whose columns are transmitted one-after-one as $\mathbf{x}(t)$ in (2), for $1 \leq t \leq T$. The code fulfills the orthogonality property $\mathbf{C}(\mathbf{s}) \mathbf{C}(\mathbf{s})^{H}=\frac{1}{n_{T}} \sum_{i=1}^{K}\left|s_{i}\right|^{2} \mathbf{I}$, which also satisfies the average power constraint assumed above. Under these assumptions, we have from [4] that the SNR and capacity of each channel use can be expressed as

$$
\mathrm{SNR}_{k}^{\text {OSTBC }}=\frac{\left\|\mathbf{H}_{k}\right\|_{F}^{2}}{n_{T}}, \quad \mathrm{C}_{k}^{\text {OSTBC }}=\frac{K}{T} \log _{2}\left(1+\frac{\left\|\mathbf{H}_{k}\right\|_{F}^{2}}{n_{T}}\right),
$$

respectively. When it comes to MRT, the principle is that the channel matrix in (2) is multiplied from the right with $\mathbf{x}(t)=$ $\mathbf{v}_{1} s(t)$, where $\mathbf{v}_{1}$ is the dominating right singular vector of $\mathbf{H}_{k}$ and $s(t)$ is the data symbol. Then, the receiver singles out the largest singular value of $\mathbf{H}_{k}$ by multiplying $\mathbf{y}_{k}(t)$ with the dominating left singular vector. The corresponding SNR and capacity of MRT is

$$
\mathrm{SNR}_{k}^{\mathrm{MRT}}=\left\|\mathbf{H}_{k}\right\|_{2}^{2}, \quad \mathrm{C}_{k}^{\mathrm{MRT}}=\log _{2}\left(1+\left\|\mathbf{H}_{k}\right\|_{2}^{2}\right),
$$

respectively [7]. It is evident that the capacity of MRT in (4) is better than that of OSTBC in (3), but one should recall that this gain comes with the requirement that the dominating right singular vector $\mathbf{v}_{1} \in \mathbb{C}^{n_{T}}$ should be fed back.

Remark 1. It is worth noting that both OSTBC and MRT are special cases of linear precoded OSTBCs [9], [10]. In this scheme, a linear precoder $\mathbf{W} \in \mathbb{C}^{n_{T} \times B}$ is used to direct the code $\mathbf{C}(\mathbf{s}) \in \mathbb{C}^{B \times T}$, that transmits $K$ symbols over $T$ slots, into advantageous spatial directions. By transmitting the columns of $\mathbf{W C}(\mathbf{s})$, the achieved SNR and capacity are

$\mathrm{SNR}_{k}^{\text {pOSTBC }}=\frac{\left\|\mathbf{H}_{k} \mathbf{W}\right\|_{F}^{2}}{B}, \mathrm{C}_{k}^{\text {pOSTBC }}=\frac{K}{T} \log _{2}\left(1+\frac{\left\|\mathbf{H}_{k} \mathbf{W}\right\|_{F}^{2}}{B}\right)$,

respectively. This general framework is capable of dealing with different kinds of channel awareness at the transmitter. When statistical CSI is available it can be exploited to allocate more power in statistically stronger spatial directions. We can then incorporate the precoder in the statistics (i.e., $\mathbf{R}_{\mathrm{Tx}, k}^{\text {new }}=\mathbf{W}^{H} \mathbf{R}_{\mathrm{Tx}, k} \mathbf{W}$ and $n_{T}^{\text {new }}=B$ ) and proceed as for OSTBCs without precoding in (3). The special case of $\mathbf{W}=\mathbf{I}$ corresponds directly to the case without precoding.

When the dominating singular vector $\mathbf{v}_{1}$ is fed back to the transmitter and used as precoder $\mathbf{W}=\mathbf{v}_{1}$ (i.e., $B=$ $K=T=1$ ), we arrive at MRT as in (4). In spatially correlated multi-user systems, it has however been shown that the directional information provided by the statistics and channel gain feedback is sufficient to achieve performance close to that of MRT [11], [12], [13].

\section{A. CDFs of Frobenius and Spectral Channel Norms}

The performance of fast-fading systems can be measured in different ways, for example, by the bit error rate (BER) or the outage capacity. Common to most measures is that they improve with increasing SNR. From (3) and (4) we see that the SNR of OSTBCs and MRT is represented by the squared Frobenius norm and squared spectral norm, respectively. In this section, we will present the cumulative distribution functions (CDFs) of these norms, under the assumption of onesided spatial correlation. These CDFs describe which values 
that the channel norms are likely to have, and they will be used extensively in the succeeding sections to develop an entropymaximizing quantization framework and to derive MMSE estimators of the SNR and the capacity.

For the purpose of describing the CDFs for the two cases of transmit-side only and receive-side only correlation in a unified way, we let $n$ denote the number of antennas at the correlated side, while $m$ denotes the number of antennas at the uncorrelated side. The distinct and strictly positive eigenvalues at the correlated side are denoted $\lambda_{1}, \ldots, \lambda_{n}$.

Using [14] and [15], the CDF of the squared Frobenius norm $\|\mathbf{H}\|_{F}^{2}$ can be expressed as

$F_{\|\mathbf{H}\|_{F}^{2}}(\rho)=1-\frac{H_{0}(\rho)}{\prod_{i=1}^{n} \lambda_{i}^{m}} \sum_{k=1}^{n} \sum_{l=1}^{m} \frac{\Psi_{k, l, m}}{\left(-\frac{1}{\lambda_{k}}\right)^{m-l+1}} e^{-\frac{\rho}{\lambda_{k}}} \sum_{j=0}^{m-l} \frac{\left(\frac{\rho}{\lambda_{k}}\right)^{j}}{j !}$,

where $H_{0}(\rho)$ is the Heaviside step function and

$$
\Psi_{k, l, m}=(-1)^{m} \sum_{i \in \Omega_{k, l}^{(n)}} \prod_{j \neq k}\left(\begin{array}{c}
i_{j}+m-1 \\
i_{j}
\end{array}\right)\left(\frac{1}{\lambda_{j}}-\frac{1}{\lambda_{k}}\right)^{-\left(m+i_{j}\right)}
$$

with $i=\left\{i_{1}, \ldots, i_{n}\right\}$ from the set $\Omega_{k, l}^{(n)}$ of all partitions of $l-1$ where the $k$ th element is zero, more formally defined as

$$
\Omega_{k, l}^{(n)} \triangleq\left\{\left\{i_{1}, \ldots, i_{n}\right\} ; \sum_{j=1}^{n} i_{j}=l-1, i_{k}=0, i_{j} \in \mathbb{Z}^{+} \forall j\right\} .
$$

The CDF of the the squared spectral norm $\|\mathbf{H}\|_{2}^{2}$ is given in [16], and the results can be summarized as

$$
F_{\|\mathbf{H}\|_{2}^{2}}(\mu)=\frac{\operatorname{det}\left[\begin{array}{c}
\mathbf{\Phi} \\
\boldsymbol{\Delta}(\mu)
\end{array}\right](-1)^{m(n-s)} H_{0}(\mu)}{\operatorname{det}(\mathbf{V}) \prod_{i=1}^{s}(m-i) !},
$$

where $t \triangleq \max (m, n), s \triangleq \min (m, n), d \triangleq \max (0, n-m)$, $\boldsymbol{\Phi} \in \mathbb{R}^{d \times n}, \boldsymbol{\Delta}(\mu) \in \mathbb{R}^{s \times n}$, and $\mathbf{V} \in \mathbb{R}^{n \times n}$. By using the lower incomplete gamma function $\gamma(a, x) \triangleq \int_{0}^{x} t^{a-1} e^{-t} d t=$ $(a-1) !\left(1-e^{-x} \sum_{k=0}^{a-1} \frac{x^{k}}{k !}\right)$, we get the expressions

$$
\begin{aligned}
& {[\boldsymbol{\Phi}]_{i j}=\left(-\frac{1}{\lambda_{j}}\right)^{n-m-i}, \quad[\mathbf{V}]_{i j}=(-1)^{n-j} \lambda_{i}^{m-n+j},} \\
& {[\boldsymbol{\Delta}(\mu)]_{i j}=\lambda_{j}^{m-i+1} \gamma\left(m-i+1, \frac{\mu}{\lambda_{j}}\right) .}
\end{aligned}
$$

Next, an entropy-maximizing quantization framework will be proposed based on the CDF of the quantized variable. Hence, the analytic expressions of the squared Frobenius norm and the squared spectral norm in (6) and (7), respectively, can be used for direct calculation of the quantization boundaries.

\section{A GENERAL ENTROPY-MAXIMIZING AND}

\section{POST-USER-SELECTION QUANTIZATION FRAMEWORK}

In this section, we consider a general framework of quantizing some variables $x_{k} \in[0, \infty)$, each with the $\operatorname{CDF} F_{k}(\cdot)$. The basic quantization property will be maximization of the information-theoretical entropy [17], and we will also consider the effect of user-selection on this type of quantization. The variables $x_{k}$ may represent the squared Frobenius or the squared spectral norm of a Rayleigh fading channel with onesided correlation (as described in the previous section), but the results in this section are valid for any continuous CDFs that fulfills $F_{k}(x)=0$, for $x_{k} \leq 0$, and $F_{k}(x)<1$, for $x_{k}<\infty$. In this paper, we generalize the quantization approach that was proposed in [18] in context of a zero-forcing precoding with limited feedback.

Quantization is the process of mapping a continuous range of values to a set $\mathcal{Q}$ of discrete values. The purpose may, for example, be that of representing a random real-valued variable with a limited number of bits so that it can be sent over a digital communication link. It is important to note that each member of $\mathcal{Q}$ represents the set of all values of the original variable that are assigned to it, and not a specific value of this set. Herein, we consider $L$-bits interval-quantization, which means that the range $[0, \infty)$ of $x_{k}$ will be divided into $2^{L}$ disjoint intervals $\left[A_{i-1}^{(k)}, A_{i}^{(k)}\right), 1 \leq i \leq 2^{L}$. Hence, the quantized variable can be fed back using $L$ bits that describe in which interval $x_{k}$ lies, while the actual value in the interval that best represents the feedback information depends on the application (e.g., estimation of $x_{k}$ or some function of it).

When designing the quantization, we need to choose the decision boundaries $A_{i}^{(k)}, 0 \leq i \leq 2^{L}$, so that some design criteria is fulfilled. There is of course no over-all optimal criteria, but from an information-theoretical perspective it makes sense to maximize the entropy of the quantization. The following lemma gives the interval-quantization of $x_{k}$ that maximizes the entropy.

Lemma 1. Let $X$ be a stochastic variable with a continuous CDF $F(x)$ that fulfills $F(x)=0$, for $x \leq 0$, and $F(x)<1$, for $x<\infty$. Assume that the sample space, $[0, \infty)$, of $X$ is quantized into $2^{L}$ disjoint intervals $\left(1 \leq i \leq 2^{L}\right)$, where the ith interval is $\left[A_{i-1}, A_{i}\right)$ with $A_{0}=0$ and $A_{2^{L}}=\infty$.

Let $\mathcal{Q}_{X} \in\left\{1, \ldots, 2^{L}\right\}$ be the quantization index (i.e., the interval in which the outcome lies) of $X$, then the quantization that maximizes the entropy of $X$ is given by

$$
A_{i}=F^{-1}\left(\frac{i}{2^{L}}\right), \quad i=1, \ldots, 2^{L}-1 .
$$

This quantization will make the outcome of $X$ equally probable in all the quantization intervals. It will also maximize the mutual information between $\mathcal{Q}_{X}$ and $g(X)$, for any invertible function $g(\cdot): \mathbb{R}^{+} \rightarrow \mathbb{R}$.

Proof: The lemma follows from a division of the CDF of $g(X)$ into $2^{L}$ disjoint intervals of equal probability.

An important result of the lemma is that even if we are interested in some function of $x_{k}$ (e.g., the capacity if $x_{k}$ is the SNR), we still only need to consider the entropy maximizingquantization in (9).

In multi-user communication, a subset of all users are selected for transmission at the same time. These users are chosen based on the CSI available at the transmitter-for example, channel statistics and feedback information. As discussed in [19], less CSI is typically required to perform user-selection than to design the actual multi-user transmission structure that 
guarantees high data throughput. The quantization given in Lemma 1 maximizes the entropy prior to user-selection, but in a multi-user system it makes more sense to design a quantizer that maximizes the post-user-selection entropy. If the variable $x_{k}$ is a measure of the channel quality (e.g., gain information), then users with large values of $x_{k}$ are more probable to be selected and we should adapt the quantization to the shift of probability mass of $x_{k}$ towards larger values.

To illustrate this principle, we propose a scheduler that takes its decision based on a combination of channel quality and fairness, but still is analytically tractable. The scheduler selects $M$ users out of $N$ based on their relative channel quality, in terms of the CDF value for the current realization of $x_{k}$. The spatial separability between users is ignored in the selection, but as shown in [20] this is actually of minor importance when the number of transmit antennas grows. The scheduler, its properties, and the resulting post-user-selection quantization are given by the following theorem.

Theorem 1. Let the channel quality of user $k$ be $x_{k} \geq 0$ and let its $C D F$ be $F_{k}(\cdot)$, for $k=1, \ldots, N$. Assume that the users are independent and that we select those $M$ users that have the largest $C D F$ values $F_{k}\left(x_{k}\right)$. Then, the post-user-selection CDFs of the channel qualities of the selected users will be $G_{M}\left(F_{k}(x)\right)$, where

$$
G_{M}(x)=\sum_{i=0}^{M-1}\left(\begin{array}{c}
N \\
i
\end{array}\right) \frac{M-i}{M} x^{N-i}(1-x)^{i} .
$$

The L-bits interval-quantization of the selected user $k$, that maximizes the post-user-selection entropy of $x_{k}$ is given by

$$
A_{i}^{(k)}=F_{k}^{-1}\left(G_{M}^{-1}\left(\frac{i}{2^{L}}\right)\right), \quad i=1, \ldots, 2^{L}-1,
$$

$A_{0}^{(k)}=0$, and $A_{2 L}^{(k)}=\infty$. This quantization will also maximize the mutual information between $\mathcal{Q}_{X}$ and $g(X)$, for any invertible function $g(\cdot): \mathbb{R}^{+} \rightarrow \mathbb{R}$.

Proof: The theorem follows from Lemma 1 and from the observations that $F_{k}\left(x_{k}\right) \in U[0,1]$ and that a selected user has any of the $l$ th largest CDFs, for $1 \leq l \leq M$, with equal probability.

An important property of the proposed scheduler is that it provides good fairness-all users are equally probable to be selected at any time. In other words, users are selected when they experience good channel conditions, based on what can be expected from each of them. It should however be noted that the user-selection proposed in Theorem 1 is idealized; in reality, the exact values of $F_{k}\left(x_{k}\right)$ are unknown at the transmitter and have to be estimated based on the available feedback information. Another observation is that when the channel statistics are identical for all users and $x_{k}$ represents the SNR, then the scheduler coincides with maximum throughput scheduling [21].

To summarize, the quantization that maximizes the preuser-selection entropy of $x_{k}$ is given in (9) of Lemma 1, while the quantization that maximizes the post-user-selection entropy is given in (11) of Theorem 1. Observe that both these expressions contain the inverse of the CDF, and we will not provide any closed-form expressions herein. Since CDFs are bijective and non-decreasing, the interval boundaries can however be calculated efficiently using a line search procedure.

\section{EXACT MMSE ESTIMATION OF SNR AND CAPACITY}

In this section, we return to the squared Frobenius and squared spectral norms of Rayleigh fading channels with one-sided correlation. We assume that the norms have been quantized in some arbitrary way (known to both the transmitter and the receiver) and fed back. Hence, the only CSI available at the transmitter is the channel statistics and that the norm can take any value in a specific interval.

Next, we introduce a framework for deriving the MMSE estimator of any function of the squared norms, given the quantized squared norm, where only a simple integral remains to be calculated. Recall from (3) and (4) that the norms exactly correspond to the SNR (at least after incorporating the factor $n_{T}$ into the correlation matrix, in the former case). Therefore, we also provide complete and exact expressions for the MMSE estimators of the SNR and the channel capacity, based on the available feedback information and statistics.

The MMSE estimators of the SNR, the capacity, and of other functions of the norm are given in Theorem 2 and Theorem 3 for the squared Frobenius norm and the squared spectral norm, respectively.

Theorem 2. Let $\rho \triangleq\|\mathbf{H}\|_{F}^{2}$ be the squared Frobenius norm of $\mathbf{H}$ and let $g(\rho)$ be an arbitrary function. If $\mathcal{Q}_{\rho}$ contains the information that $A \leq \rho<B$ (with $A \leq B$ ), then the conditional MMSE estimator of $g(\rho)$ is

$$
E\left\{g(\rho) \mid \mathcal{Q}_{\rho}\right\}=\frac{C}{\prod_{i=1}^{n} \lambda_{i}^{m}} \sum_{k=1}^{n} \sum_{l=1}^{m} \frac{\Psi_{k, l, m} G_{m-l, k}(A, B)}{(-1)^{m-l+1}(m-l) !},
$$

where $C^{-1}=F_{\|\mathbf{H}\|_{F}^{2}}(B)-F_{\|\mathbf{H}\|_{F}^{2}}(A)$ is calculated using (6) and

$$
G_{m-l, k}(A, B)=\int_{A}^{B} g(\rho) \rho^{m-l} e^{-\frac{\rho}{\lambda_{k}}} d \rho .
$$

$$
\text { If } g(\rho)=\rho \text { or } g(\rho)=\log _{2}(1+\rho) \text {, then we have }
$$

$$
\begin{aligned}
& G_{N, k}(A, B)=\left[-\frac{(N+1) !}{\left(\frac{1}{\lambda_{k}}\right)^{N+2}} e^{-\frac{\rho}{\lambda_{k}}} \sum_{i=0}^{N+1} \frac{\left(\frac{\rho}{\lambda_{k}}\right)^{i}}{i !}\right]_{A}^{B} \\
& G_{N, k}(A, B)=\sum_{l=0}^{N}\left(\begin{array}{c}
N \\
l
\end{array}\right) \frac{l ! \lambda_{k}^{l+1}}{(-1)^{N-l+1}}\left[\frac{e^{-\frac{\rho}{\lambda_{k}}}}{\log _{e}(2)} \sum_{i=1}^{l} \frac{1}{i} \sum_{j=0}^{i-1} \frac{\left(\frac{\rho+1}{\lambda_{k}}\right)^{j}}{j !}\right. \\
&\left.+\frac{e^{\frac{1}{\lambda_{k}}}}{\log _{e}(2)} E_{1}\left(\frac{1+\rho}{\lambda_{k}}\right)+\log _{2}(1+\rho) e^{-\frac{\rho}{\lambda_{k}}} \sum_{i=0}^{l} \frac{\left(\frac{\rho+1}{\lambda_{k}}\right)^{i}}{i !}\right]_{A}^{B}
\end{aligned}
$$

respectively, where $E_{1}(\rho)=\int_{\rho}^{\infty} \frac{e^{-u}}{u} d u$ is the exponential integral and the notation $[F(\rho)]_{A}^{B}=F(B)-F(A)$ was used.

Proof: The theorem follows from straightforward integration of the PDF of $\|\mathbf{H}\|_{F}^{2}$ (the CDF was given in (6)). 
Theorem 3. Let $\mu \triangleq\|\mathbf{H}\|_{2}^{2}$ be the squared spectral norm of $\mathbf{H}$ and let $g(\mu)$ be an arbitrary function. If $\mathcal{Q}_{\mu}$ contains the information that $A \leq \mu<B$ (with $A \leq B$ ), then the conditional MMSE estimator of $g(\mu)$ is

$$
\begin{gathered}
E\left\{g(\mu) \mid \mathcal{Q}_{\mu}\right\}=C \sum_{\alpha \in \mathcal{A}_{n}} \frac{\prod_{k=d+1}^{n} \lambda_{\alpha_{k}}^{t-k+1} \prod_{k=m-s+1}^{m}(k-1) !}{(-1)^{\operatorname{per}(\alpha)} \prod_{k=1}^{d}\left(-\lambda_{\alpha_{k}}\right)^{d-k}} \\
\times \sum_{l=1}^{s}(-1)^{l} \sum_{\beta \in \mathcal{B}_{l, s}} \sum_{k=0}^{K_{l}(\beta)} \sum_{\tilde{k} \in \widetilde{\Omega}_{k}^{(l)}} \frac{1}{\tilde{k}_{1} ! \cdots \tilde{k}_{l} !} \frac{\widetilde{G}_{k, \beta}(A, B)}{\prod_{i=1}^{l} \lambda_{\alpha_{\left(\beta_{i}+d\right)}}^{\tilde{k}_{i}}},
\end{gathered}
$$

where $C^{-1}=\operatorname{det}\left[\begin{array}{c}\mathbf{\Phi} \\ \boldsymbol{\Delta}(B)\end{array}\right]-\operatorname{det}\left[\begin{array}{c}\mathbf{\Phi} \\ \boldsymbol{\Delta}(A)\end{array}\right]$ is calculated using (8) and $\alpha=\left\{\alpha_{1}, \ldots, \alpha_{n}\right\} \in \mathcal{A}_{n}$, where $\mathcal{A}_{n}$ is the set of all permutations of $\{1, \ldots, n\}$ and $(-1)^{\operatorname{per}(\alpha)}$ is the sign of $\alpha$ (i.e., positive for even and negative for odd permutations). $\beta=\left\{\beta_{1}, \ldots, \beta_{l}\right\} \in \mathcal{B}_{l, s}$, where $\mathcal{B}_{l, s}$ is the set of all subsets of $\{1, \ldots, s\}$ that fulfill $\beta_{1}<\ldots<\beta_{l}$, and $K_{l}(\beta)=\sum_{i=1}^{l} m-\beta_{i} . \tilde{k}=\left\{\tilde{k}_{1}, \ldots, \tilde{k}_{l}\right\} \in \widetilde{\Omega}_{k}^{(l)}$, where $\widetilde{\Omega}_{k}^{(l)}$ is the set of all partitions of $k$ that satisfy $0 \leq \tilde{k}_{i} \leq m-\beta_{i}$, and is defined as

$$
\widetilde{\Omega}_{k}^{(l)} \triangleq\left\{\left\{\tilde{k}_{1}, \ldots, \tilde{k}_{l}\right\} ; \sum_{j=1}^{k} \tilde{k}_{j}=k, 0 \leq \tilde{k}_{j} \leq m-\beta_{j} \forall j\right\} .
$$

Finally, with $D_{\beta} \triangleq\left(\sum_{i=1}^{l} \lambda_{\alpha_{\left(\beta_{i}+d\right)}}^{-1}\right)^{-1}$, we have that

$$
\widetilde{G}_{k, \beta}(A, B)=\int_{A}^{B} g(\mu) e^{-\frac{\mu}{D_{\beta}}}\left(k \mu^{k-1}-\frac{1}{D_{\beta}} \mu^{k}\right) d \mu .
$$

If $g(\mu)=\mu$ or $g(\mu)=\log _{2}(1+\mu)$, then we have

$$
\widetilde{G}_{k, \beta}(A, B)=\left[e^{-\frac{\mu}{D_{\beta}}}\left(\mu^{k+1}+\frac{k !}{D_{\beta}^{-(k+1)}} \sum_{i=0}^{k} \frac{\left(\frac{\mu}{D_{\beta}}\right)^{i}}{i !}\right)\right]_{A}^{B},
$$$$
\widetilde{G}_{k, \beta}(A, B)=\left[\frac{e^{\frac{1}{D_{\beta}}}(-1)^{k}}{\log _{e}(2)} E_{1}\left(\frac{1+\mu}{D_{\beta}}\right)+\frac{\log _{2}(1+\mu) e^{-\frac{\mu}{D_{\beta}}}}{(-1)^{k}}\right.
$$$$
\left.+\sum_{i=1}^{k}\left(\begin{array}{c}
k \\
i
\end{array}\right) \frac{e^{-\frac{\mu}{D_{\beta}}}}{(-1)^{k-i}}\left(\frac{\log _{2}(1+\mu)}{(1+\mu)^{-i}}+\frac{(i-1) !}{D_{\beta}^{-i} \log _{e}(2)} \sum_{j=0}^{i-1} \frac{\left(\frac{1+\mu}{D_{\beta}}\right)^{j}}{j !}\right)\right]_{A}^{B},
$$

respectively, where $E_{1}(\mu)=\int_{\mu}^{\infty} \frac{e^{-u}}{u} d u$ is the exponential integral and the notation $[F(\rho)]_{A}^{B}=F(B)-F(A)$ was used.

Proof: The theorem follows from an expansion of the determinant in the numerator of (7), similar to that in [22, Theorem 2.29], differentiation, and integration.

The expressions in Theorem 2 and Theorem 3 can be used directly to estimate the SNR and the capacity in an MMSE sense. The potential gains, when combined with the entropy-maximizing quantization of the previous section, will be illustrated numerically in the next section.

\section{Numerical Results}

In this section, we will illustrate the gain of taking the channel statistics and the post-user-selection distribution into account when designing the channel gain quantization for
OSTBC and MRT systems. We consider the downlink of a circular cell with radius $R$ around an elevated base station. There are eight active mobile users and these are uniformly distributed in the the area $0.1 R \leq r \leq R$. The base station is equipped with a four-antenna uniform circular array (UCA) with half a wavelength antenna separation and experiences no local scattering, while the users are exposed to rich scattering and are equipped with two antennas each. The environment is thus modeled as Rayleigh fading with correlation at the transmitter side and the angular spread (i.e., the standard deviation) of the Gaussian distributed scatterers at the user side is assumed to be 15 degrees (as seen from the base station). The signal power is assumed to decay as $1 / r^{4}$ and the mean SNR (with random transmit and receive beamformers) experienced by a user at the cell boundary is $10 \mathrm{~dB}$.

The CDF of the average cell throughput over different communication scenarios has been simulated. Each of the 15000 scenarios represents a unique setup with randomly positioned mobiles and constant channel statistics. The average cell throughput of a scenario is calculated over 80 channel realizations, where each is used for transmission to a single user using either an OSTBC (with coding rate 3/4) or MRT (with perfect feedback of the directional information). Four different feedback/scheduling approaches are compared:

- Pre-user-selection quantization, where the gain information (squared Frobenius or squared spectral norm) is quantized to maximize the pre-user-selection entropy (Lemma 1), the user with the largest MMSE estimate of its CDF is selected, and the achievable throughput is estimated using Theorem 2 or 3 and reduced with a multiplicative factor to fit an outage probability of $5 \%$.

- Post-user-selection quantization, designed as the previous approach except that the post-user-selection distribution is taken into account in the quantization (Theorem 1).

- The lower bound, where the quantization is based on an uncorrelated system (with the same average power) and the transmission rate is estimated conservatively based on the lower boundary of the quantization interval.

- The upper bound, corresponding to the capacity achieved when the channel gain information is fed back exactly.

The CDFs of the cell throughput of these four approaches are given in Fig. 1(a) (with OSTBC) and Fig. 1(b) (with MRT) with 3 and 5 bits of feedback per user (i.e., increasing performance), or with exact feedback as an upper bound. It have previously been shown numerically in [18] that these numbers of bits are enough to achieve most or all of the feedback gain, and the same observation is valid here; the proposed system achieves $95 \%$ of the capacity with 3 bits of feedback and $99 \%$ with 5 bits. It is clear that quantization based on the post-user-selection distribution leads to a slight throughput improvement, which also can be seen as a way of decreasing the error probability for a fixed transmission rate.

It is also evident that the use of statistic-dependent quantization (as proposed herein) can enhance the performance considerably, in comparison with keeping the quantization 
fixed over time (as in the lower bound). In other words, the implementational simplicity of having fixed quantization levels comes with a non-negligible performance degradation when comparing with a scheme that changes the levels with the slowly varying channel statistics.

As expected, the throughput with MRT is much higher than with OSTBC, but it should be pointed out that the selected user in MRT needs to feed back additional directional information [7] and that the performance of OSTBC can be improved in this transmit-side-correlated environment by using linear precoding (see Remark 1).

\section{CONCLUSION}

In this paper, two independent but related concepts of wireless MIMO communication systems with limited feedback have been considered. First, a general quantization framework was presented for the purpose of feedback. The key design features were to maximize the entropy of the feedback variable and to take the post-user-selection distribution into account in the quantization. The latter is of particular importance when feeding back channel gain information, since strong users are more probable to be scheduled and therefore have a greater need of getting a fine quantization.

Next, MMSE estimation of arbitrary functions of both the squared Frobenius norm and the squared spectral norm was considered. These norms correspond directly to the SNR of systems based on the commonly studied techniques of OSTBCs and MRT, respectively, and measure the performance of many other systems. An MMSE framework was introduced, conditioned on an arbitrarily quantized channel norm, and exact expressions were presented for estimation of the SNR and the capacity. Finally, the features of these two concepts (adapting the quantization to the statistics and robust performance estimation) were illustrated numerically.

\section{ACKNOWLEDGMENT}

The authors would like to acknowledge useful discussions with David Hammarwall.

\section{REFERENCES}

[1] G. J. Foschini and M. J. Gans, "On limits of wireless communications in a fading environment when using multiple antennas," Wireless Personal Commun., vol. 6, pp. 311-335, 1998.

[2] E. Telatar, "Capacity of multi-antenna Gaussian channels," European Trans. Telecom., vol. 10, pp. 585-595, 1999.

[3] E. Björnson and B. Ottersten, "Pilot based Bayesian channel norm estimation in Rayleigh fading multi-antenna systems," in Proc. Nordic Conf. Radio Science and Commun. (RVK'08), 2008.

[4] V. Tarokh, H. Jafarkhani, and A. Calderbank, "Space-time block codes from orthogonal designs," IEEE Trans. Inf. Theory, vol. 45, pp. 14561467, 1999.

[5] E. Larsson and P. Stoica, Space-Time Block Coding for Wireless Communications. Cambridge University Press, 2003.

[6] T. Lo, "Maximum ratio transmission," IEEE Trans. Commun., vol. 47, pp. 1458-1461, 1999.

[7] D. Love, R. Heath, and T. Strohmer, "Grassmannian beamforming for multiple-input multiple-output wireless systems," IEEE Trans. Inf. Theory, vol. 49, pp. 2735-2747, 2003.

[8] R. Ertel, P. Cardieri, K. Sowerby, T. Rappaport, and J. Reed, "Overview of spatial channel models for antenna array communication systems," IEEE Personal Commun. Mag., vol. 5, pp. 10-22, 1998.

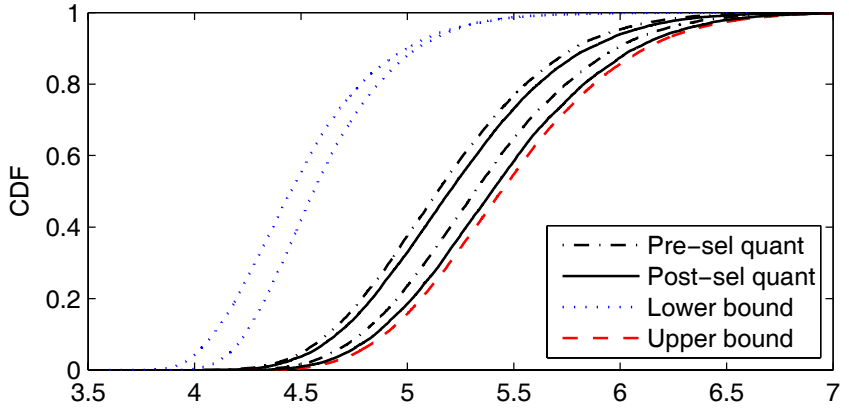

(a) Cell throughput with OSTBCs (rate 3/4) [bits/symbol].

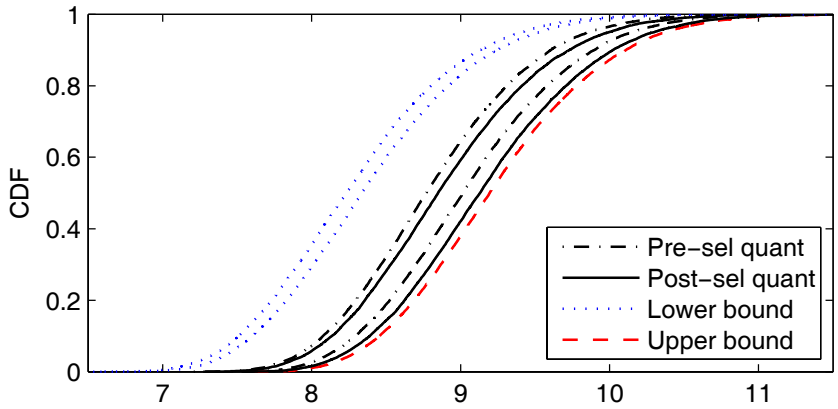

(b) Cell throughput with MRT (exact directional information) [bits/symbol].

Fig. 1. The cumulative distribution functions (CDFs) of the average cell throughput achieved for the four different scheduling/feedback approaches and with either 3 or 5 bits of channel gain feedback per user (increasing performance), or with exact feedback as an upper bound.

[9] G. Jöngren, M. Skoglund, and B. Ottersten, "Combining beamforming and orthogonal space-time block coding," IEEE Trans. Inf. Theory, vol. 48, pp. 611-627, 2002.

[10] E. Larsson, G. Ganesan, P. Stoica, and W. Wong, "On the performance of orthogonal space-time block coding with quantized feedback," IEEE Commun. Lett., vol. 6, pp. 487-489, 2002.

[11] D. Hammarwall, M. Bengtsson, and B. Ottersten, "Acquiring partial CSI for spatially selective transmission by instantaneous channel norm feedback," IEEE Trans. Signal Process., vol. 56, pp. 1188-1204, 2008.

[12] E. Björnson, D. Hammarwall, and B. Ottersten, "Beamforming utilizing channel norm feedback in multiuser MIMO systems," in Proc. IEEE SPAWC'07, 2007.

[13] D. Hammarwall, M. Bengtsson, and B. Ottersten, "Utilizing the spatial information provided by channel norm feedback in SDMA systems," IEEE Trans. Signal Process., to be published.

[14] E. Scheuer, "Reliability of an $m$-out-of- $n$ system when component failure induces higher failure rates in survivors," IEEE Trans. Rel., vol. 37, pp. 73-74, 1988.

[15] S. Amari and R. Misra, "Closed-from expressions for distribution of sum of exponential random variables," IEEE Trans. Rel., vol. 46, pp. 519-522, 1997.

[16] M. Kang and M. Alouini, "Impact of correlation on the capacity of MIMO channels," in Proc. IEEE ICC'03, vol. 4, 2003, pp. 2623-2627.

[17] T. Cover and J. Thomas, Elements of Information Theory. Wiley, 1991.

[18] E. Björnson and B. Ottersten, "Exploiting long-term statistics in spatially correlated multi-user MIMO systems with quantized channel norm feedback," in Proc. IEEE ICASSP'08, 2008.

[19] R. Zakhour and D. Gesbert, "A two-stage approach to feedback design in multi-user MIMO channels with limited channel state information," in Proc. IEEE PIMRC'07, 2007.

[20] X. Zhang, E. Jorswieck, and B. Ottersten, "User selection schemes in multiple antenna broadcast channels with guaranteed performance," in Proc. IEEE SPAWC'07, 2007.

[21] R. Knopp and P. Humblet, "Information capacity and power control in single-cell multiuser communications," in Proc. IEEE ICC'95, 1995.

[22] M. McKay, "Random matrix theory analysis of multiple antenna communication systems," Ph.D. dissertation, University of Sydney, 2006. 